\title{
Traveling waves for a nonlocal dispersal SIR epidemic model with the mass action infection mechanism
}

\author{
Xin $\mathrm{Wu}^{1}$ and Zhaohai $\mathrm{Ma}^{2}$ \\ ${ }^{1}$ East China Jiao Tong University \\ ${ }^{2}$ China University of Geosciences Beijing
}

July 8, 2021

\begin{abstract}
This paper is concerned with a nonlocal dispersal susceptible-infected-recovered (SIR) epidemic model adopted with the mass action infection mechanism. We mainly study the existence and non-existence of traveling waves connecting the infection-free equilibrium state and the endemic equilibrium state. The main difficulties lie in the fact that the semiflow generated here does not admit the order-preserving property. Meanwhile, this new model brings some new challenges due to the unboundedness of the nonlinear term. We overcome these difficulties to obtain the boundedness of traveling waves with the speed $\$ \mathrm{c}>\mathrm{c}_{-}\{\backslash$ min $\} \$$ by some analysis techniques firstly and then prove the existence of traveling waves by employing Lyapunov-LaSalle theorem and Lebesgue dominated convergence theorem. By utilizing a approximating method, we study the existence of traveling waves with the critical wave speed $\$ c_{-}\{\backslash \min \} \$$. Our results on this new model may provide some implications on disease modelling and controls.
\end{abstract}

\section{Hosted file}

wuxin.pdf available at https://authorea.com/users/424510/articles/529579-traveling-wavesfor-a-nonlocal-dispersal-sir-epidemic-model-with-the-mass-action-infection-mechanism 